\title{
Four Points Function Fitted and First Derivative Procedure for Determining the End Points in Potentiometric Titration Curves: Statistical Analysis and Method Comparison
}

\author{
Sherif A. KHOLEIF \\ Alexandria University, Faculty of Science, Chemistry Department, Horreya Avenue, \\ P. O. Box 426, Ibrahimia, Alexandria 21321, Egypt
}

\begin{abstract}
A new method that belongs to the differential category for determining the end points from potentiometric titration curves is presented. It uses a preprocess to find first derivative values by fitting four data points in and around the region of inflection to a non-linear function, and then locate the end point, usually as a maximum or minimum, using an inverse parabolic interpolation procedure that has an analytical solution. The behavior and accuracy of the sigmoid and cumulative non-linear functions used are investigated against three factors. A statistical evaluation of the new method using linear least-squares method validation and multifactor data analysis are covered. The new method is generally applied to symmetrical and unsymmetrical potentiometric titration curves, and the end point is calculated using numerical procedures only. It outperforms the "parent" regular differential method in almost all factors levels and gives accurate results comparable to the true or estimated true end points. Calculated end points from selected experimental titration curves compatible with the equivalence point category of methods, such as Gran or Fortuin, are also compared with the new method.
\end{abstract}

(Received December 25, 2000; Accepted March 22, 2001)

\section{Introduction}

For determining the inflection point or end point in potentiometric titration curves by the "parent" of the differential category, the regular first derivative (RFD) or second derivative (RSD) method is simple and straight forward, but lacks the accuracy of the end point calculated under the influence of certain factors. This category of methods depends on the presence of an inflection point to function properly, but does not require any prior information about the nature of the analyte. The end point can be located in this case if two sequential titrant additions result in a change of at least $0.3 \mathrm{pH}$ unit $(17.7 \mathrm{mV}$ at $25^{\circ} \mathrm{C}$ ) in the titration curve. Programmable versions of the RFD and RSD methods locate the end point using inverse parabolic interpolation $^{1}$ and Neville inverse interpolation ${ }^{2}$ algorithms, respectively. Variable deviations of the calculated end point using the RFD method in comparison with the true end point arise from a plot of $\mathrm{d} y / \mathrm{d} x(x=\mathrm{ml}$ of titrant and $y=$ response; either $\mathrm{mV}$ or $\mathrm{pH})$ against the quantity $\left(x_{i+1}+x_{i}\right) / 2$ or the mean of two successive titrant volume additions, as described by Gran. ${ }^{3}$ A similar situation is also found with the RSD method.

The equivalence point category of methods are usually specific for the type of analyte and/or shape of titration curve. In most cases, they involve a derived equation for each analyte and titration types considered without the necessity of the presence of an inflection point in the titration curve. The Gran's plot $^{3}$ is one example known since 1950. A more elaborate discussion involving a statistical analysis about the advantages and limitations of this method can be found in the literature. ${ }^{4}$ The functions of Gran's method, using $\mathrm{pH}$ or $\mathrm{mV}$ reading, and

E-mail: ShKh@Link.net in-depth coverage of the calculations involved ${ }^{5}$ as well as the uncertainty of the equivalence point ${ }^{6}$ may be found in the literature. The case to handle other simple types of one-step precipitation, complexometric and redox titrations is reported. ${ }^{7}$ Each type of analyte and titration requires a specific derived function based on prior knowledge about the stoichiometry of the reactants in a way that the method cannot be easily generalized. In the same equivalence point category, Fortuin's method $^{8}$ is generally applied to symmetrical potentiometric titration curves with equidistant titrant volumes in the region of inflection, and is based on a derived equation. The method uses four data points in the region of inflection to calculate two parameters related to the transition in this region. The limitations and conditions were also stated $^{8}$ in relation to calculations of the two parameters.

In the present work, a new method with a programmable algorithm is presented. It is demonstrated here using the sigmoid and cumulative functions implemented in the algorithms sigmoid first derivative (SFD) and cumulative first derivative (CFD) and compared together with the results obtained from the RSD method as the "parent" of the differential category. The work also includes graphical and statistical analyses for the three tested procedures covering linear least-squares method validation and multifactor analysis. The new method maintains the wide application of the second derivative method and its independence on the nature of analyte and type of titration. At the same time, it is generally applied to symmetrical and unsymmetrical titration curves with more accurate results for the calculated end point. The end points calculated from selected experimental titration curves are also compared with the Gran and Fortuin methods. At this stage, a preliminary treatment related to the problem of non-equidistant titrant volume addition is introduced. 


\section{Experimental}

\section{Reagents}

A KOH titrisol ampoule, $\mathrm{HCl}$, nitrilotriacetic acid (NTA), $\mathrm{KCl}$ and $\mathrm{KNO}_{3}$ were all analytical grade reagents obtained from Merck-Germany. Potassium hydrogen phthalate was analytical grade from Riedel-de Haën and ethylenediamine from Aldrich.

\section{Preparation of solutions}

Standard 0.100 M KOH was prepared from a titrisol ampoule, checked by titration using $0.0500 \mathrm{M}$ potassium hydrogen phthalate and used as a titrant. Standard $0.118 \mathrm{M} \mathrm{HCl}$ was prepared from a concentrated reagent, standardized by titration against standard $\mathrm{KOH}$ and used as a titrant. Standard $\mathrm{HCl}$ test solutions were prepared by dilution from a standard stock solution and adjusted to a total ionic strength $I=0.1(\mathrm{KCl})$. The test solutions were further standardized by titration against standard KOH. NTA sample solutions were prepared by dilution from a saturated solution and adjusted to a total ionic strength $I=1\left(\mathrm{KNO}_{3}\right)$. An ethylenediamine sample solution was prepared by diluting a given weight of the reagent and without any supporting electrolyte for ionic strength adjustment. All solutions were prepared in double distilled water, which was also degassed for preparing the $\mathrm{KOH}$ standard.

\section{Instrumentation and apparatus}

The potentiometric titration system consisted of a $100 \mathrm{ml}$ or $10 \mathrm{ml}$ covered thermostated glass cell kept at $25.0^{\circ} \mathrm{C}$ and fitted with inlets for nitrogen gas, a burette tip immersed into the test solution and a combined glass electrode or glass electrode and a salt bridge connected to an external calomel reference electrode. The calomel electrode and salt bridge were filled with the same supporting electrolyte and kept at the same ionic strength of the measured solution. The nitrogen gas was saturated with $0.1 \mathrm{M}$ $\mathrm{KCl}$ or $1 \mathrm{M} \mathrm{KNO}_{3}$, depending on the titration system used, and allowed to evolve in the space above the measured solution. The electrode was connected to an Orion SA720 potentiometer and a Metrohm Dosimat 665 motor burette $(1 \mathrm{ml}$ or $10 \mathrm{ml}$ capacity) was used to deliver the standard titrant solution. Both the burette and potentiometer were connected to a portable PC and the titration was monitored using the TIT212 data acquisition and titration control software. A 40.0 or $5.00 \mathrm{ml}$ volume of the test or sample solutions was titrated automatically. If the titration curve is solely used for end point(s) determination with the new method, data in the region(s) of inflection of the curve are only required. Moreover, an ionic strength adjustment of the test or sample solution is not necessary in this case, but it is preferred to use a combined electrode or reference electrode with an internal filling solution $\geq 2 \mathrm{M} \mathrm{KCl}$.

\section{Results and Discussion}

\section{Functions and algorithm for end points determination}

The new method uses one non-linear function, either the sigmoid or cumulative, to fit four data points in the region of inflection of the titration curve. The choice of the four data points is achieved by locating the maximum variation (maximum $\mathrm{d} y / \mathrm{d} x$ ) between two successive titrant volumes (two data points) in addition to the two adjacent data points bracketing the maximum variation region. Once the coefficients of the function are obtained, the first derivative of that function $\left(y^{\prime}\right)$ is calculated for the original titrant volumes $(x)$ in the region of inflection. Three first derivative data points at three original titrant volumes thus form a parabola with either a maximum or minimum, depending on the response $(y)$. The maximum or minimum of the parabola, i.e., the calculated end point $\left(x_{\mathrm{e}}\right)$, can be directly calculated using an inverse parabolic interpolation equation ${ }^{1}$ for this type of data.

The sigmoid function (Eq. (1)) and its first derivative (Eq. (2)) used and implemented in the SFD algorithm are as follows:

$$
\begin{aligned}
& y=a+b /\left[1+\mathrm{e}^{(c-x) / d}\right], \\
& y^{\prime}=\mathrm{d} y / \mathrm{d} x=\left[b \mathrm{e}^{(c-x) / d}\right] /\left[\left(1+\mathrm{e}^{(c-x) / d}\right)^{2} d\right],
\end{aligned}
$$

while the cumulative function (Eq. (3)) and its first derivative (Eq. (4)) implemented in the CFD algorithm are as follows:

$$
\begin{aligned}
& y=a+0.5 b\{1+\operatorname{erf}[(x-c) /(\sqrt{2} d)]\}, \\
& y^{\prime}=\mathrm{d} y / \mathrm{d} x=0.5\left\{b \mathrm{e}^{-0.5\left[(x-c)^{2} / d^{2}\right]} \sqrt{2}\right\} /\{\sqrt{\pi} d\} .
\end{aligned}
$$

The term "erf" given in Eq. (3) stands for "error function of", which can be calculated using a programmable algorithm. Coefficients $a, b, c$ and $d$ of Eqs. (1) and (3) are the start of transition, height of transition, center of transition (a value close to the end point) and transition steepness respectively. They can be obtained by applying a common simplex algorithm ${ }^{1}$ or using a preprogrammed form. ${ }^{9}$ The first derivative data are then calculated using Eq. (2) or (4) and a selection of three points forming a parabola among them (with either a maximum or minimum) is performed; the end point is found within the range of those points and calculated directly from the analytical solution of an inverse parabolic interpolation, ${ }^{1}$ as follows:

$$
x_{\mathrm{e}}=x_{2}-\frac{1}{2} \frac{\left(x_{2}-x_{1}\right)^{2}\left(y_{2}^{\prime}-y_{3}^{\prime}\right)-\left(x_{2}-x_{3}\right)^{2}\left(y_{2}^{\prime}-y_{1}^{\prime}\right)}{\left(x_{2}-x_{1}\right)\left(y_{2}^{\prime}-y_{3}^{\prime}\right)-\left(x_{2}-x_{3}\right)\left(y_{2}^{\prime}-y_{1}^{\prime}\right)},
$$

where $x_{\mathrm{e}}$ is the calculated end point and $x_{1}, x_{2}, x_{3}, y_{1}^{\prime}, y_{2}^{\prime}$ and $y_{3}^{\prime}$ are the titrant volumes and their corresponding first derivatives, respectively. The condition to choose the three points forming the parabola is that $y_{2}^{\prime}$ be greater or less than both $y_{1}^{\prime}$ and $y_{3}^{\prime}$. Thus, the real computational work is limited to finding the coefficients of either Eq. (1) or (3), whereas the remaining SFD or CFD algorithm involves straight forward calculations. The major limitation ${ }^{3}$ of the RSD method for calculating the first derivatives $(\mathrm{d} y / \mathrm{d} x)$ at values of $\left(x_{i+1}+x_{i}\right) / 2$ is now overcome in the new method.

\section{Testing the accuracy of the new method by simulation}

Three factors were found to affect the differential methods that depend solely on the shape of the titration curve to calculate an end point from the region of inflection of that curve. Those factors are the size of transition, the interval or increment of titrant addition $(\Delta)$ in the region of inflection and a less common factor is the position or location of the true end point $\left(v_{\mathrm{e}}\right)$ within two successive titrant additions at the maximum variation region ( $\mathrm{d} y / \mathrm{d} x$ is maximum). To test the behavior of Eqs. (1) and (3) and the accuracy of the new algorithms (SFD and CFD) and to simultaneously avoid random and systematic errors and easily set the factors limits, the necessary data $(\mathrm{ml}, \mathrm{pH})$ were simulated according to the following conditions. The analyte is either a strong acid or acid with $\mathrm{p} K_{\mathrm{a}}$ of 3,5 and $7\left(K_{\mathrm{a}}\right.$ is the acid dissociation constant). $\mathrm{p} K_{\mathrm{a}}$ is used only to simulate titration curves with variable transition sizes (first factor), and contributes as a meaningful numerical quantity in the factorial design analysis given later. The second factor $(\Delta)$ is studied at 


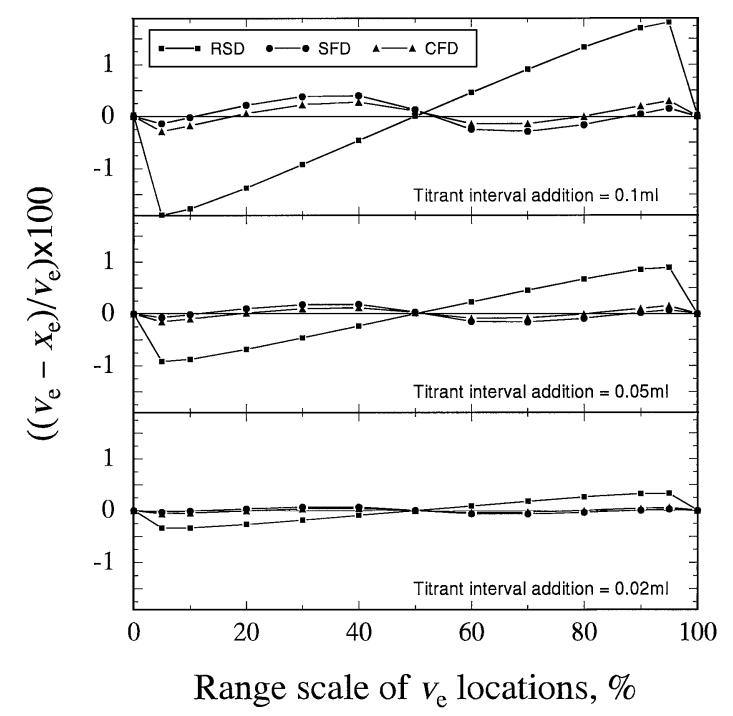

Fig. 1 Percentage residual errors relative to the true values $\left(v_{\mathrm{e}}\right)$ as a function of the position or location of the true end point within two successive titrant additions in the region of inflection as percent range scale at constant $\mathrm{p} K_{\mathrm{a}}$ factor of 3 and different intervals of titrant addition $(\Delta)$.

$0.02,0.05$ and $0.1 \mathrm{ml}$ at all factors levels and at constant conditions of $0.1 \mathrm{M}$ strong base titrant and $50 \mathrm{ml}$ initial volume of acid. A change in the initial volume to $5 \mathrm{ml}$ at the same conditions requires a ten times smaller $\Delta$ value to obtain equivalent results, which can be verified from the quantity $\left(v_{\mathrm{e}} / \Delta\right)$. The position of the true end point $\left(v_{\mathrm{e}}\right.$; the third factor) is varied within the interval of two successive titrant additions (maximum $\mathrm{d} y / \mathrm{d} x$ ) at constant positions in increments of $10 \%$ relative to the $\Delta$ value, except at the boundaries where the increment was $5 \%$ relative. This is equivalent to range scale ${ }^{10}$ the interval between 0 and 1 and multiply the new scale by 100 . The range scale allows a comparison at different factors levels, since the lengths of the three $\Delta$ values used $(0.02,0.05$ and 0.1 $\mathrm{ml}$ ) are not equal. If for instance $\Delta=0.02 \mathrm{ml}$ in the region of inflection that is between 1.98 and $2.00 \mathrm{ml}$, the 0,50 and $100 \%$ positions of $v_{\mathrm{e}}$ (percentage range scale of $v_{\mathrm{e}}$ ) are at 1.98, 1.99 and $2.00 \mathrm{ml}$ respectively. The variation in the position of the true end point within the titrant interval is achieved by changing the concentration of the acid to a maximum of 1, 2.5 and $4.8 \%$ for $\Delta$ values of $0.02,0.05$ and $0.1 \mathrm{ml}$, respectively. However, the number of data points used in the investigation of this third factor is constant and at the same relative positions. The simulated titration data for the weak acid are obtained from the equation

$$
\begin{aligned}
& {[\mathrm{H}]^{3}+[\mathrm{H}]^{2}\left\{\left[K_{\mathrm{a}}+\left(C_{\mathrm{b}} x\right) /\left(v_{0}+x\right)\right]\right\}} \\
& -[\mathrm{H}]\left\{K_{\mathrm{a}}\left[C_{\mathrm{b}}\left(v_{\mathrm{e}}-x\right) /\left(v_{0}+x\right)\right]+K_{\mathrm{w}}\right\}-K_{\mathrm{a}} K_{\mathrm{w}}=0,
\end{aligned}
$$

where $[\mathrm{H}], C_{\mathrm{b}}, v_{\mathrm{o}}$, and $K_{\mathrm{w}}$ are the hydrogen ion equilibrium concentration, the concentration of the base titrant, the initial volume of acid and the ion product constant of water, respectively.

A multifactor comparison among the three methods (RSD, SFD and CFD) of the same category was carried out using the results obtained from the simulated data. The aim of this comparison was to test each method and function behavior for its accuracy over multifactor multilevel conditions without the interference of random and systematic errors resulting from the experimental measurements that may possibly alter the

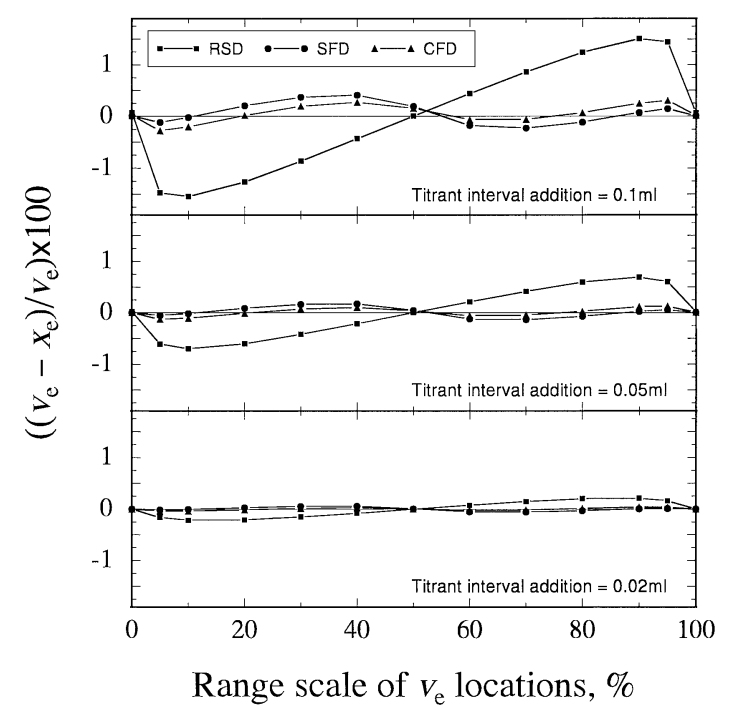

Fig. 2 Percentage residual errors relative to the true values $\left(v_{\mathrm{e}}\right)$ as a function of the position or location of the true end point within two successive titrant additions in the region of inflection as percent range scale at constant $\mathrm{p} K_{\mathrm{a}}$ factor of 5 and different intervals of titrant addition $(\Delta)$.

functions behavior, i.e., investigating a pure method error.

The percentage residual errors between the true end points $\left(v_{\mathrm{e}}\right)$ and the calculated end points $\left(x_{\mathrm{e}}\right)$ were calculated at all factors levels. Two-dimension graphical representations of the results are given in Figs. 1 and 2 at the $\mathrm{p} K_{\mathrm{a}}$ factor levels of 3 and 5 , respectively.

The third factor was found to influence the accuracy of the calculated end point $\left(x_{\mathrm{e}}\right)$, as shown in Figs. 1 and 2. The effect of this factor is less pronounced for the newly introduced methods than the RSD method at almost all factor levels. From the graphical representations of Figs. 1 and 2, all methods calculate an $x_{\mathrm{e}}$ value with very good accuracy at levels of 0,50 and $100 \%$. However, the levels in between those values, that were not previously investigated, produce lower accuracy depending on the other two factors. The percentage residual errors of the new methods are lower and better distributed. The same behavior in Fig. 1 was also obtained in the strong acid case, but with a slightly higher percentage of residual errors. From Figs. 1 and 2, the SFD algorithm was found to give more accurate results near to the boundaries of the interval of two successive titrant additions; this behavior is similar at all factors levels, and occurs at constant positions. Thus, for optimum results in general, SFD is recommended for calculating the end point at less than $14 \%$ or more than $86 \%$ ve position, while CFD is used for the positions in between those limits. The approximate position of the end point can be determined while locating the maximum variation region at start (at maximum $\mathrm{d} y / \mathrm{d} x$ ), by applying RFD or RSD to get an approximate value, or directly from the parameter " $c$ " of Eq. (1) or (3).

The equivalence point category of methods were not included in Figs. 1 and 2 because their derived functions assume an ideal titration type and/or symmetrical titration curve following in most cases the Nernst equation, in which case, the calculated end point from a simulated curve will be the same as the true end point by definition. However, a better comparison can be made with experimental results, which will be given later in the text.

\section{Method validation for results obtained by simulation}

The true end points or reference values ( $v_{\mathrm{e}}$; free from random 
Table 1 Results of the least-squares method validation between the reference (true) end point $v_{\mathrm{e}}$ and the test (calculated) end point $x_{\mathrm{e}}$ for the three methods at different factors levels

\begin{tabular}{|c|c|c|c|c|c|c|}
\hline \multirow{2}{*}{ Parameter } & \multicolumn{3}{|c|}{$\mathrm{p} K_{\mathrm{a}}=3, \Delta=0.02 \mathrm{ml}$} & \multicolumn{3}{|c|}{$\mathrm{p} K_{\mathrm{a}}=5, \Delta=0.02 \mathrm{ml}$} \\
\hline & RSD & SFD & CFD & RSD & SFD & CFD \\
\hline$a^{\prime}$ & $1.1 \pm 0.2$ & $-0.04 \pm 0.07$ & $0.08 \pm 0.06$ & $0.7 \pm 0.2$ & $0.04 \pm 0.06$ & $0.07 \pm 0.04$ \\
\hline$b^{\prime}$ & $0.46 \pm 0.01$ & $1.02 \pm 0.04$ & $0.96 \pm 0.03$ & $0.66 \pm 0.08$ & $1.02 \pm 0.03$ & $0.96 \pm 0.02$ \\
\hline$r^{\prime}$ & 0.774 & 0.994 & 0.995 & 0.928 & 0.996 & 0.998 \\
\hline$F$ & 16.4 & 838 & 1177 & 68.0 & 1368 & 2917 \\
\hline$t_{\mathrm{cal}\left(a^{\prime}\right)}$ & 4.66 & -0.611 & 1.54 & 4.21 & -0.831 & 2.10 \\
\hline$t_{\mathrm{cal}\left(b^{\prime}\right)}$ & 4.05 & 29.0 & 34.3 & 8.25 & 37.0 & 54.0 \\
\hline$t_{b^{\prime}}^{\prime}$ & 2.80 & 0.627 & 1.48 & 3.00 & 0.852 & 2.03 \\
\hline \multirow[t]{3}{*}{$\mathrm{SSR} \times 10^{5}$} & 8.7 & 0.82 & 0.52 & 4.3 & 0.51 & 0.21 \\
\hline & \multicolumn{3}{|c|}{$\mathrm{p} K_{\mathrm{a}}=3, \Delta=0.1 \mathrm{ml}$} & \multicolumn{3}{|c|}{$\mathrm{p} K_{\mathrm{a}}=5, \Delta=0.1 \mathrm{ml}$} \\
\hline & RSD & SFD & CFD & RSD & SFD & CFD \\
\hline$a^{\prime}$ & $1.2 \pm 0.2$ & $-0.04 \pm 0.07$ & $0.08 \pm 0.06$ & $1.0 \pm 0.2$ & $-0.03 \pm 0.07$ & $0.10 \pm 0.05$ \\
\hline$b^{\prime}$ & $0.4 \pm 0.1$ & $1.02 \pm 0.04$ & $0.96 \pm 0.03$ & $0.5 \pm 0.1$ & $1.02 \pm 0.03$ & $0.95 \pm 0.03$ \\
\hline$r^{\prime}$ & 0.709 & 0.993 & 0.995 & 0.811 & 0.994 & 0.996 \\
\hline$F$ & 11.1 & 768 & 1057 & 21.1 & 897 & 1258 \\
\hline$t_{\mathrm{cal}\left(a^{\prime}\right)}$ & 4.69 & -0.597 & 1.26 & 4.61 & -0.497 & 1.85 \\
\hline$t_{\mathrm{cal}\left(b^{\prime}\right)}$ & 3.34 & 27.7 & 32.5 & 4.59 & 29.9 & 35.5 \\
\hline$t_{b^{\prime}}^{\prime}$ & 2.75 & 0.562 & 1.33 & 2.84 & 0.606 & 1.78 \\
\hline $\mathrm{SSR} \times 10^{5}$ & 257 & 22.4 & 14.5 & 195 & 19.1 & 11.9 \\
\hline
\end{tabular}

The uncertainty of the parameters corresponds to one standard deviation. The parameters $a^{\prime}, b^{\prime}$ are those of the equation $x_{\mathrm{e}}=\mathrm{a}^{\prime}+b^{\prime} v_{\mathrm{e}} ; t_{\mathrm{cal}}=$ (parameter value/parameter error). SSR $=$ sum of squares residuals. $t_{\text {crit }}=t_{11,95 \%}=2.201 . t_{b^{\prime}}^{\prime}=\left[\left(b^{\prime}-1\right) /\left(1-r^{2}\right)^{1 / 2}\right][N-2]^{1 / 2} ; N=13$.

and systematic errors) are compared with those calculated $\left(x_{\mathrm{e}}\right)$ using the three differential methods by linear least-squares and determine whether the calculated values are significantly different from $v_{\mathrm{e}}$. The test also detects the bias, the type of errors involved and the validity of each method.

A comparison was carried out at the factors levels $\mathrm{p} K_{\mathrm{a}}=3$ and 5 and $\Delta$ values of 0.02 and $0.1 \mathrm{ml}$. The linear least-squares coefficients of the comparison test are given in Table 1 together with some statistical quantities for evaluating the linear fit (explained in the footnote of the table). The correlation coefficient values $\left(r^{\prime}\right)$ given in Table 1 show, in general, that both SFD and CFD are much less affected by the multifactor multilevel conditions compared with the RSD method. The fit is also evaluated from the test statistic $F$ values, in which its application was previously discussed. ${ }^{11}$ The intercept $\left(a^{\prime}\right)$ for the new method was not significantly different from zero as found from the $t_{\mathrm{cal}\left(\mathrm{a}^{\prime}\right)}$ values that are less than the $t$-student critical value $t_{\text {crit }}=t_{11,95 \%}$ (for 11 degrees of freedom and $95 \%$ confidence) indicating the absence of constant systematic errors associated with SFD and CFD. At the same time, the values of their slopes $\left(b^{\prime}\right)$ were significant $\left(t_{\text {cal(b') }}>t_{\text {crit }}\right)$, but not significantly different from 1 or the ideal slope $\left(t_{b^{\prime}}^{\prime}<t_{\text {crit }}\right)$, indicating the absence of proportional systematic errors as well. ${ }^{12}$ Thus, both SFD and CFD are useful for calculating end points over multifactor multilevel conditions. On the other hand, the test shows that RSD is affected by both constant and proportional systematic errors under the same conditions, thus affecting the accuracy of the calculated end point. The $t^{\prime} b^{\prime}$ and $t_{\text {cal }\left(a^{\prime}\right)}$ for SFD are lower than CFD, even though the overall fitting parameters for CFD are slightly better (higher $r^{\prime}, F$ and lower SSR). This is because of the residual $\left(v_{\mathrm{e}}-x_{\mathrm{e}}\right)$ at the boundaries of two successive titrant additions ( $v_{\mathrm{e}}$ range scale position is $14 \%$ on each side) is lower in case of SFD, as given earlier.

\section{Factorial design analysis for the residual errors}

In this test, the amount of dependence of the three differential methods (RSD, SFD and CFD) on the factors levels is investigated. A non-central composite design comprises the size of the transition (represented by $\mathrm{p} K_{\mathrm{a}}$ ) and the interval of titrant addition $(\Delta)$, which are considered to be the independent variables, while the mean of the absolute residual errors is taken as the response $\left(y_{\mathrm{re}}\right)$ at each factor combination $\left(\mathrm{p} K_{\mathrm{a}}=3,5,7 ; \Delta\right.$ $=0.02,0.05$ and $0.1 \mathrm{ml}$ ). The resulting multifactor model equation is

$$
y_{\mathrm{re}}=a_{0}+a_{1} \cdot \mathrm{p} K_{\mathrm{a}}+a_{2} \cdot \Delta+a_{12} \cdot \mathrm{p} K_{\mathrm{a}} \cdot \Delta+a_{11} \cdot \mathrm{p} K_{\mathrm{a}}^{2}
$$

and its coefficients are given in Table 2. Since all of the factors levels investigated were the same for each method, it is possible to compare the effect of each factor and factor interaction on that particular method. From the coefficients in Table 2, the $\Delta$ factor is the most significant in all three methods with the coefficient $a_{2}$ for RSD being about six times as large as that in the other two methods. The factor interaction $\left(\mathrm{p} K_{\mathrm{a}} \cdot \Delta\right)$ is significant in RSD and the dependence of this method on the factor interaction and the other factors is about ten times more than the other two methods for which those factors are almost insignificant, as given in Table 2. The offset parameter $\left(a_{\mathrm{o}}\right)$ is much lower for the SFD and CFD than the RSD method, which is in accordance with the least-squares pairwise comparison discussed earlier. As a conclusion from this test, both SFD and CFD are much less affected by the size of the transition and the $\Delta$ factors and factor combination than RSD, which make them suitable for calculating the end points at different factors levels with good accuracy.

The investigation made by simulating over a multifactor multilevel conditions, especially for the first two factors created titration curves with transition sizes ranging from $\sim 0.3 \mathrm{pH}$ unit $(\sim 17 \mathrm{mV})$ to $\sim 5 \mathrm{pH}$ units $(\sim 295 \mathrm{mV})$ and $v_{\mathrm{e}} / \Delta \geq 20$; this range is 
Table 2 Coefficients of Eq. (7) for the three methods using the original variables data and the response $\left(y_{\mathrm{re}}\right)$ is taken as the mean of the absolute residual errors ${ }^{\mathrm{a}}$

\begin{tabular}{cccc}
\hline Coefficient & RSD & SFD & CFD \\
\hline$a_{0}$ & $-0.007 \pm 0.003$ & $-0.0010 \pm 0.0007$ & $-0.0007 \pm 0.0007$ \\
$a_{1}$ & $0.003 \pm 0.001$ & $0.0004 \pm 0.0003$ & $0.0003 \pm 0.0003$ \\
$a_{2}$ & $0.28 \pm 0.02$ & $0.044 \pm 0.005$ & $0.037 \pm 0.005$ \\
$a_{12}$ & $-0.021 \pm 0.005$ & $-0.002 \pm 0.001$ & $-0.0016 \pm 0.0009$ \\
$a_{11}$ & $-0.0004 \pm 0.0001$ & $-0.00004 \pm 0.00003$ & $-0.00003 \pm 0.00003$ \\
$r^{\prime}$ & 0.997 & 0.995 & 0.995 \\
\hline
\end{tabular}

a. The mean of the absolute residual errors $=\left(\frac{1}{N} \sum\left|\nu_{\mathrm{e}}-x_{\mathrm{e}}\right|\right) ; N=9$.

Table 3 Calculated end points from experimental strong acid-base titration curves at different factors levels of $\Delta$ and variable percentage position of the estimated true value $\left(\boldsymbol{v}_{\mathrm{e}}^{\prime}\right)$. The percentage absolute relative residual errors are given in brackets beneath the end points. The initial volume is $40.0 \mathrm{ml},[\mathrm{HCl}]=5.180 \times 10^{-3} \mathrm{M}$ or $5.108 \times 10^{-3} \mathrm{M}$ and $[\mathrm{KOH}]=0.100 \mathrm{M}$ in $I=0.1(\mathrm{KCl})$ at $25.0^{\circ} \mathrm{C}$.

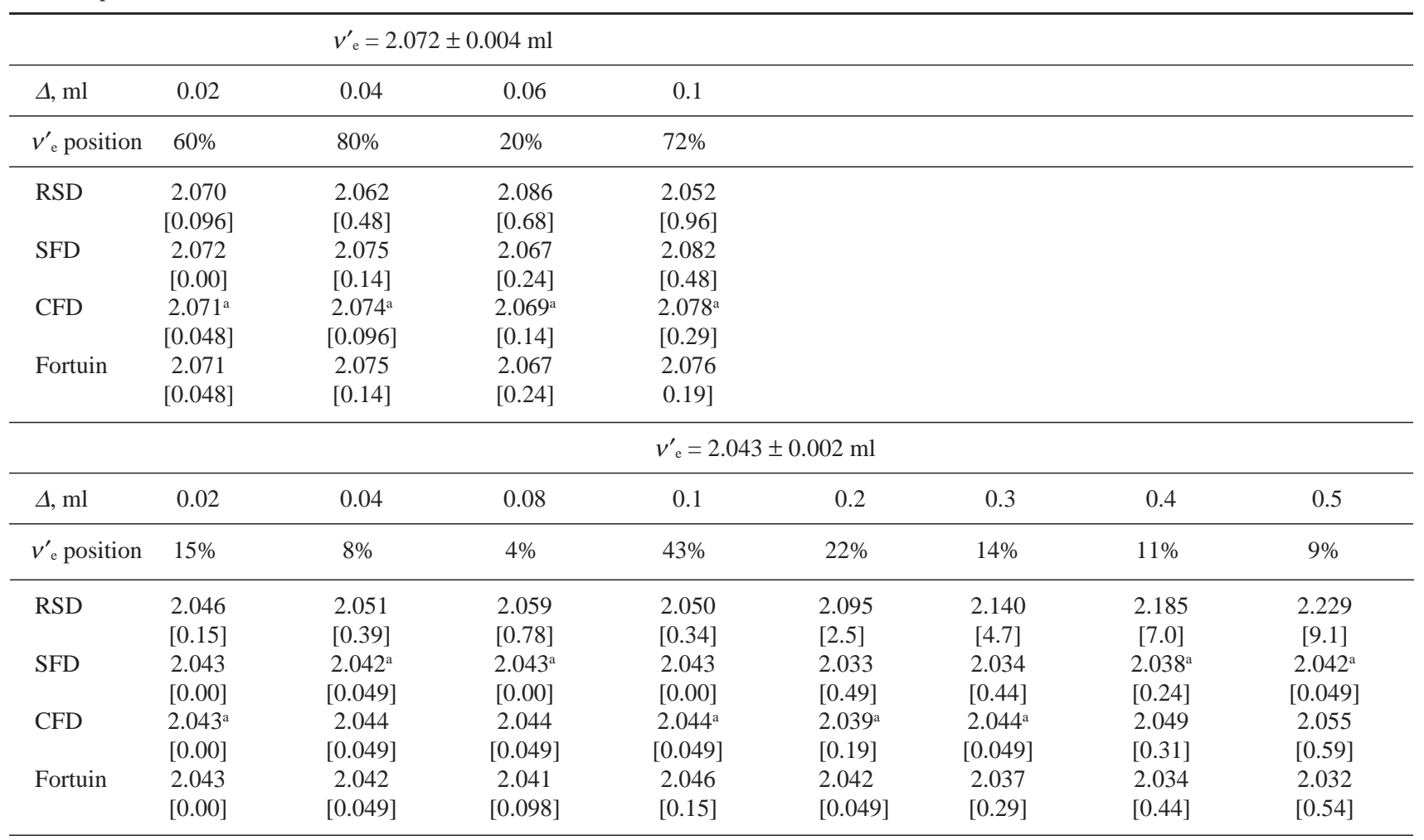

The uncertainties are the $\pm 95 \%$ confidence. a. Recommended procedure based on the position of the end point (see text).

useful for most practical applications using the SFD and CFD algorithms.

\section{Application of SFD and CFD to experimental data}

Table 3 demonstrates the calculated end points $\left(x_{\mathrm{e}}\right)$ of two experimental strong acid-base titration curves using the three differential methods and Fortuin's equivalence point method. The end points were calculated at four different factor levels $(\Delta)$, each corresponding to a different percentage position of the estimated true end point $\left(v_{\mathrm{e}}^{\prime}\right)$ within two successive titrant additions at the inflection region (the third factor). The effects of the second and third factors on the experimental end points calculated are very close to the previously found effects shown in Figs. 1 and 2 and obtained using the simulated data. The slight deviation from these effects is attributed to the presence of random experimental errors. The calculated end points using the SFD and CFD algorithms are close to the estimated true end points $(2.072 \pm 0.004$ and $2.043 \pm 0.002 \mathrm{ml}$; uncertainties are the $\pm 95 \%$ confidence) than the RSD method.
The equivalence points of the two experimental titration curves tested were also calculated using Gran's method, and were found to be $2.069 \pm 0.005 \mathrm{ml}\left(v_{\mathrm{e}}^{\prime}=2.072 \pm 0.004 \mathrm{ml}\right)$ and $2.037 \pm 0.005 \mathrm{ml}\left(v_{\mathrm{e}}^{\prime}=2.043 \pm 0.002 \mathrm{ml}\right)$. These values were calculated using the theoretical Nernst slope $(59.16 \mathrm{mV}$ at $25^{\circ} \mathrm{C}$ ). A relatively small variation in the value of the Nernst slope will produce a significant change in the calculated equivalence point value.

The equivalence point values calculated using Fortuin's method and given in Table 3 are close to the end point values calculated using the SFD and CFD methods. In these particular examples of Table 3 , the quantity necessary to calculate the equivalence point using the Fortuin's method was obtained numerically using a computer software rather than the nomograph, according to the original publication of 1961, which will slightly increase the error of the equivalence point. Thus, a variation in $\Delta$ accompanied by a slight departure from the ideal symmetrical shape and a decrease in the size of the transition of the titration curve affect the calculated equivalence 
point value using the Fortuin's method. This is partly demonstrated in Table 3 for the equivalence point values in the range $0.5 \geq \Delta \geq 0.2$, for which a random variation is noticed with increasing $\Delta$ from 0.1 to $0.5 \mathrm{ml}$. The SFD or CFD algorithm still shows good accuracy at higher $\Delta$ and lower $v_{\mathrm{e}} / \Delta$ values than those investigated in this work. The $x_{\mathrm{e}}$ values are also affected by an increase in $\Delta$ in a different manner than those of Fortuin's method. More accurate end point values are obtained when using both SFD and CFD, depending on the position of the end point in the region of inflection, as given earlier.

Five milliliters from each of three NTA samples were titrated against $0.100 \mathrm{M} \mathrm{KOH}$. The mean of the end points calculated using the CFD method was $0.2174 \mathrm{ml}$, identical to the value found by Fortuin's method $(0.2174 \mathrm{ml})$ and close to that from the RSD method $(0.2172 \mathrm{ml})$ when $\Delta=0.002 \mathrm{ml}$ and $v_{\mathrm{e}} / \Delta=$ 109. Increasing $\Delta$ to $0.01 \mathrm{ml}\left(v_{\mathrm{e}} / \Delta=22\right)$, the CFD, Fortuin and RSD values were $0.2178,0.2178$ and $0.2154 \mathrm{ml}$, respectively. The accuracy of the RSD method was affected when $\Delta$ was increased, as stated earlier.

A $40.0 \mathrm{ml}$ of ethylenediamine sample was titrated against $0.118 \mathrm{M} \mathrm{HCl}$ with a non-equidistant titrant volume addition and a minimum of $0.1 \mathrm{ml}$ acid in the region of inflection. The CFD and RSD end points were found to be 12.878 and $12.882 \mathrm{ml}$, respectively.

A sample of the experimental precipitation titration data is given in Fortuin's publication ${ }^{8}$ with $\Delta=0.5 \mathrm{ml}$ and $v_{\mathrm{e}} / \Delta=10$. The Fortuin's equivalence point value found was $5.006 \mathrm{ml}$ against a value of $5.003 \mathrm{ml}$, calculated using the CFD $\left(x_{\mathrm{e}}\right.$ position is $40 \%$ ). An example for a strong acid-base titration produced with the same interval of $0.5 \mathrm{ml}$ had a Fortuin's equivalence point of $2.956 \mathrm{ml}$ against a value of $2.952 \mathrm{ml}$, calculated using the SFD method ( $x_{\mathrm{e}}$ position is $90 \%$ ).

The new method was also tested successfully on experimental data with different shapes of titration curves including di- and polyprotic acids and bases, acidimetric and alkalimetric titration of ligands and metals, precipitation titration and weight titration of copper and EDTA using copper-selective electrode replacing titrant volumes by weights. ${ }^{13}$ Furthermore, it is possible to sequentially apply the method to locate multiple end points in the same curve.

\section{Preliminary treatment for non-equidistant titrant volumes}

Despite the fact that non-equidistant titrant volume addition is not a restrictive problem facing the practical application in potentiometry, it would be an extra advantage to consider it toward the generalization of a given method.

SFD and CFD algorithms do not hinder finding an end point in case of non-equidistant titrant volume addition, though the amount of the estimated relative error and the behavior of the functions are not absolutely known at this stage. The error is expected to decrease as the size of the transition is diminished. The probability of distributing non-equidistant titrant volume intervals in the region of inflection is very large to treat, as in Figs. 1 and 2. However, at this stage, the case of the strong acid-base given in Table 3 is considered. The interval of titrant addition between the two data points producing the maximum variation, i.e., maximum $\mathrm{d} y / \mathrm{d} x$, are varied from 0.02 to $0.1 \mathrm{ml}$ so that the four points are distributed as follows: (1.94, 2.04, $2.06,2.12)$; (1.94, 2.04, 2.08, 2.14); (1.94, 2.04, 2.10, 2.16) and $(1.94,2.04,2.14,2.20)$. The coefficients of SFD or CFD are calculated from Eq. (1) or (3), while the first derivative is calculated from Eq. (2) or (4), as previously given, to know which three points form the parabola. If the interval at the maximum $\mathrm{d} y / \mathrm{d} x$ for instance is $0.02 \mathrm{ml}$ and the three points forming the parabola are $1.94,2.04,2.06$, Eq. (2) or (4) is directly used to calculate (interpolate) a first derivative for the point $(2.02 \mathrm{ml})$ between 1.94 and $2.04 \mathrm{ml}$ to be equidistant with 2.04 and $2.06 \mathrm{ml}$. Thus the interval of the two points at maximum $\mathrm{d} y / \mathrm{d} x$ dictates the necessary new value required to reach an equidistant arrangement. The values of the end point found using this technique for the four distributions given above are in order: $\mathrm{CFD}=2.043, \mathrm{SFD}=2.042, \mathrm{SFD}=2.043, \mathrm{CFD}=$ 2.044. This is just a good preliminary treatment for the problem at this stage.

\section{Conclusion}

The new differential category method is used for the determination of end points from symmetrical and unsymmetrical potentiometric titration curves with good accuracy. The method is purely numerical and can be implemented using PC software. It does not require any prior information about the chemical nature of the species involved in a titration, and a calibration of the potentiometer is not required. It is readily applicable when a titration curve with one or more inflection points exist, each with a transition size $\geq 0.3 \mathrm{pH}$ unit or $\geq 17.7 \mathrm{mV}$ at $25^{\circ} \mathrm{C}$ so that the SFD or CFD treats each inflection region one at a time. Thus, it may be applied to titration curves of mono-, di- and polyprotic acids and bases, complexometry, precipitation and redox. The extra advantage of the SFD or CFD algorithm over the equivalence point class, apart from the earlier discussion, is that it can be modified with new functions, treatments and improved to consider new requirements. A preliminary solution to the problem of nonequidistant titrant volume addition is available through the current method presented at this stage and further investigation and improvement will be considered.

\section{References}

1. W. H. Press, S. A. Teukolsky, W. T. Vetterling, and B. P. Flannery, "Numerical Recipes", 1992, Cambridge University Press, 213, 395, 402.

2. H. R. Schwarz, "Numerical Analysis", 1989, John Wiley \& Sons Ltd., Chichester, New York, Brisbane, Toronto, Singapore, 114.

3. G. Gran, Anal. Chim. Acta, 1988, 206, 111.

4. L. M. Schwartz, Anal. Chim. Acta, 1989, 225, 205.

5. F. J. C. Rossotti and H. Rossotti, J. Chem. Educ., 1965, 7, 375.

6. L. M. Schwartz, J. Chem. Educ., 1992, 69, 879.

7. C. Maccà and G. G. Bombi, Analyst, 1989, 114, 463.

8. J. M. H. Fortuin, Anal. Chim. Acta, 1961, 24, 175.

9. K. Ebert, H. Ederer, and T. L. Isenhour, "Computer Applications in Chemistry", 1989, VCH Verlagsgesellschaft, Weinheim, 390.

10. M. A. Sharaf, D. L. Illman, and B. R. Kowalski, "Chemometrics", 1986, Vol. 82, "Chemical Analysis", John Wiley \& Sons, New York, Chichester, Brisbane, Toronto, Singapore, 193.

11. G. Anderegg and S. Kholeif, Talanta, 1994, 41, 1507.

12. D. L. Massart, B. G. M. Vandeginste, S. N. Deming, Y. Michotte, and L. Kaufman, "Chemometrics: a Textbook", 1988, Vol. 2, "Data Handling in Science and Technology", Elsevier Science Publishers, Amsterdam, Oxford, New York, Tokyo, 53.

13. E. Nakamura, Y. Nagahara, and H. Namiki, Anal. Sci., 1991, 7 (supplement), 753. 